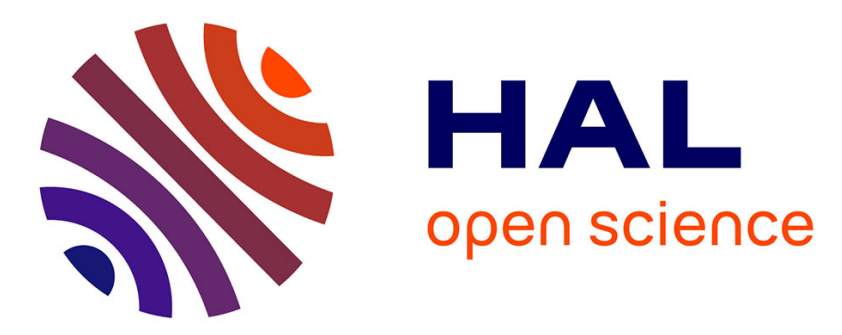

\title{
Hydromechanical modelling of an initial boundary value problem: Studies of non-uniqueness with a second gradient continuum
}

\author{
F Marinelli, Y. Sieffert, R. Chambon
}

\section{- To cite this version:}

F Marinelli, Y. Sieffert, R. Chambon. Hydromechanical modelling of an initial boundary value problem: Studies of non-uniqueness with a second gradient continuum. Springer International Publishing Switzerland 2015, May 2014, Hong Kong, China. hal-02004541

HAL Id: hal-02004541

https://hal.science/hal-02004541

Submitted on 1 Feb 2019

HAL is a multi-disciplinary open access archive for the deposit and dissemination of scientific research documents, whether they are published or not. The documents may come from teaching and research institutions in France or abroad, or from public or private research centers.
L'archive ouverte pluridisciplinaire HAL, est destinée au dépôt et à la diffusion de documents scientifiques de niveau recherche, publiés ou non, émanant des établissements d'enseignement et de recherche français ou étrangers, des laboratoires publics ou privés. 


\title{
Hydromechanical modelling of an initial boundary value problem: Studies of non- uniqueness with a second gradient continuum
}

\author{
F. Marinelli, Y. Sieffert, R. Chambon \\ Grenoble Université Joseph Fourier, Laboratoire 3S-R, UJF, G-INP, CNRS, B.P. 53X, 38041 \\ Grenoble Cedex France
}

\begin{abstract}
A non-uniqueness study for a hydromechanical boundary value problem is performed. A fully saturated porous medium is considered using an elastoplastic constitutive equations to describe the mechanical behavior of the skeleton. A real hydromechanical experiment which consists in a hollow cylinder test on a Boom Clay sample is modelled. It is shown that the time step discretisation of the numerical problem has an effect on the initialisation of the Newton-Raphson algorithm on a given time step. Different solutions for the same initial boundary value problem can consequently be found.
\end{abstract}

\section{Introduction}

In the simulation of initial boundary value problems using constitutive equations for geomaterial behavior, it is well known that some difficulties can arise, particularly if degradation of the materials occurs. These problems have been studied for the case of single phase materials and some theoretical results have been established. Within the small strain assumption and for a rate problem, the uniqueness can be proved using the so-called Hill exclusion functional [1]. From a numerical standpoint, in case of non-uniqueness, the solution can be influenced by different numerical inputs such as the spatial discretization of the problem, the size of the time step, the mesh spacing, some tolerance values and so on (see [2]).

In this study, a real hydromechanical experiment is modelled using a finite element code Lagamine (University of Liège). A local hydromechanical second gradient model (see [3]) is considered to obtain regularised solutions of this initial boundary value problem. No other regularization is necessary in terms of the fluid phase or the solid-fluid coupling.

We show that, changing the time discretisation of the numerical problem, different solutions can be found. In function of the shear bands path during the time, a classification of all computed solutions is proposed. 


\section{Local hydromechanical second gradient model}

It is well known that classical continuum medium cannot be considered to model the localization of plastic strains. An internal length has to be introduced into the model in order to obtain mesh independent solutions of an initial boundary value problem. In this respect, the problem can be considered regularised. The regularization (that means the introduction of an internal length into the model) cannot restore the uniquess of the solution as it is proved in [4] for a local second gradient continuum model.

In the framework of microstructure continuum [5], a local hydromechanical second gradient model can be considered [3]. The problem is solved by enforcing (in a weak form) the balance equations for the mixture (equation 1), and the mass conservation for the fluid (equation 2). Both equations are written in the current solid configuration denoted $\Omega^{t}$ (updated Lagrangian configuration).

$\int_{\Omega^{t}}\left(\sigma_{i j}^{t} \frac{\partial u_{i}^{*}}{\partial x_{j}^{t}}+\Sigma_{i j k}^{t} \frac{\partial^{2} u_{i}^{*}}{\partial x_{j}^{t} \partial x_{k}^{t}}\right) d \Omega^{t}=\int_{\Omega^{t}} \rho^{m i x, t} g_{i} u_{i}^{*} d \Omega^{t}+\int_{\Gamma_{\sigma}^{t}}\left(\bar{t}_{i} u_{i}^{*}\right) d \Gamma^{t}$,

$\int_{\Omega^{t}}\left(\dot{M}^{t} p^{*}-m_{i}^{t} \frac{\partial p^{*}}{\partial x_{i}^{t}}\right) d \Omega^{t}=\int_{\Omega^{t}} Q^{t} p^{*} d \Omega^{t}-\int_{\Gamma_{q}^{t}} \bar{q}^{t} p^{*} d \Gamma^{t}$

In equation (1), Terzaghi's principle of effective stress is assumed to compute the Cauchy stress of the mixture $\sigma_{i j}^{t}$. The mass density of the mixture at the time $t$ $\rho^{\text {mix,t }}$ is defined as follows:

$\rho^{m i x, t}=\rho^{s}\left(1-\phi^{t}\right)+\rho^{w, t} \phi^{t}$,

where $\rho^{s}$ is the solid phase density (assumed to be incompressible, i.e., $\rho^{s}$ is a constant); $\rho^{w, t}$ is the fluid density and $\phi^{t}$ is the porosity defined as $\phi^{t}=\Omega^{w, t} / \Omega^{t}$. $\Omega^{t}$ is the current volume of a given mass of the solid skeleton and $\Omega^{w, t}$ the corresponding volume of fluid. The fluid is assumed to be compressible. All variables appearing in equations (1) and (2) are explained as follows:

- $u_{i}^{*}$ is any kinematically admissible virtual displacement field.

- $\Sigma_{i j k}^{t}$ is the double stress dual of the virtual second micro kinematic gradient.

- $x_{i}$ is the current coordinate.

- $g_{i}$ is the gravity acceleration.

- $\bar{t}_{i}$ is the external (classical) force per unit area.

- $m_{i}^{t}$ is the mass flow of water, which is governed by Darcy's law.

- $\dot{M}^{t}$ is the time derivative of the fluid mass of the skeleton.

- $p^{*}$ is any kinematically admissible virtual pore pressure.

- $Q^{t}$ is a sink term.

- $\Gamma_{q}^{t}$ is the part of the boundary where the input fluid mass per unit area $\bar{q}^{t}$ is prescribed 
To solve the equations (1) and (2) at time $\tau=t+\Delta t$ with a Newton-Rapshon algorithm a consistent linearisation of the system has to be performed. All the developments of the linearization for a local hydromechanical second gradient model are detailed in [3].

The classical part of the constitutive equation, giving the effective stress of the skeleton, is described by a non-associated elasto-plastic model based on the Drucker-Prager yield field (Plasol model, [6]). Cohesion $c$ and friction angle for a compressive triaxial path $\varphi$, represent the hardening/softening variables for this elasto-plastic constitutive equation. The only internal variable that characterizes the hardening/softening behavior of the material is the Von Mises equivalent plastic strain $\varepsilon_{e q}^{p}$, which describes degradation due to the shear. Dilatancy is considered as a constant during plastic flow. A hyperbolic variation of the friction angle and cohesion is proposed as hardening law. The elastic behavior is modelled by isotropic linear elasticity. This constitutive equations is computed with a return mapping algorithm [7].

The other part (giving the double stress) is a particular case of a more general isotropic linear relation derived by [8] that involves five independent parameters, in the interests of simplicity only one parameter (namely $\mathrm{D}$, see [9]) is used.

\section{Non-uniqueness studies}

In order to perform our non-uniqueness studies, we use realistic material properties related to the behavior of Boom Clay [10]. In our study we choose to model a real hydromechanical hollow cylinder experiment performed in Lausanne [11]. This experiment is divided in two phases, that are described in figure 1. A non-uniqueness study is carried out for Plasol constitutive equations by considering phase A of boundary conditions that represents a hydromechanical unloading. In phase B, the evolution of the shear bands with time is modelled. All computations presented are performed under plane strain hypothesis to model the behavior of a horizontal section of the hollow cylinder.

From a numerical point of view, non-uniqueness can be studied by changing the initialization of the Newton-Raphson algorithm for a given time step. In our case, the initialization of the numerical algorithm is obtained from the nodal velocity field $v_{j}^{n-1}$ (that one of the previous step) at the first iteration of the current step.

$x_{j}^{n^{i=1}}=x_{j}^{n-1}+v_{j}^{n-1} \Delta t$,

where $\Delta t$ represents the step size, $x_{j}^{n-1}$ is the nodal coordinate at the previous step and $x_{j}^{n^{i=1}}$ is the nodal coordinate for the first iteration of the current step. Different sequences of time steps can cause a change in the initialisation of the numerical problem for each time step. In this way, non-uniqueness can be studied for the same initial boundary value. 


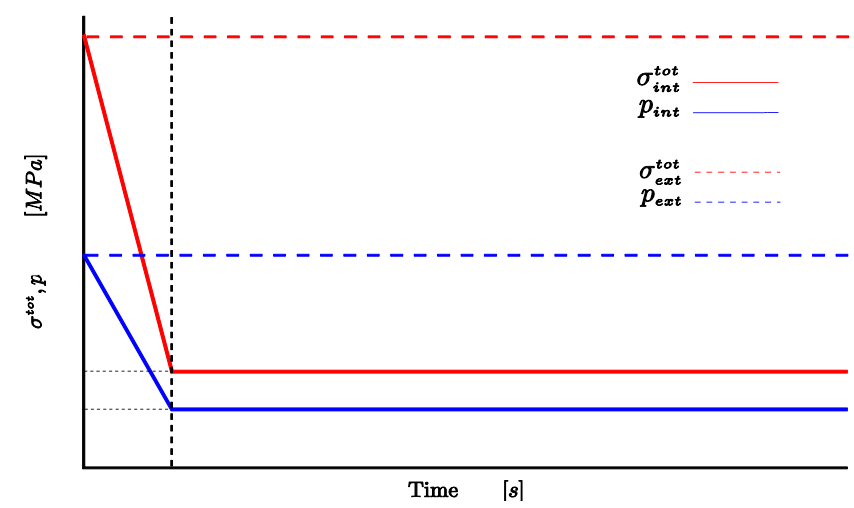

Fig. 1.The initial boundary conditions on the inner and external side of the hollow cylinder: a continous line represents boundary conditions on the inner hollow cylinder, a dot line on the external hollow cylinder.

In order to visualize the non-uniqueness of this initial boundary value problem, the evolution of the shear strains are shown in terms of the loading index. This quantity is defined as follows: if a Gauss point undergoes plastic loading, a small blue square is plotted, otherwise (in the case of elastic unloading or reloading of the Gauss point), a small yellow square is plotted.

Basically, all the localized deformation patterns present two kinds of shear band configurations: the first is characterized by some crossing shear bands (the socalled conjugated shear bands), while the second is characterized by spiral shear bands (the so-called periodic shear bands).

Among the great number of computations performed five solutions, which are representative of the non-uniqueness problem, are shown in figure 2 . All the solutions are presented in Figure 3 and can be classified qualitatively according to shear bands evolution as follows:

- Computations with radial symmetry ISO-0.

- Computations with shear bands that start from a thin plastified region around the inner hollow cylinder, which then grow towards the external part of the sample (solutions ISO-1 and ISO-4).

- Computations with shear bands that appear by an elastic unloading in a thick plastified zone of the hollow cylinder at the end of phase A (solutions ISO-2 and ISO-3).

The development of the shear bands during the phase B of the boundary conditions is shown in figure 3. 


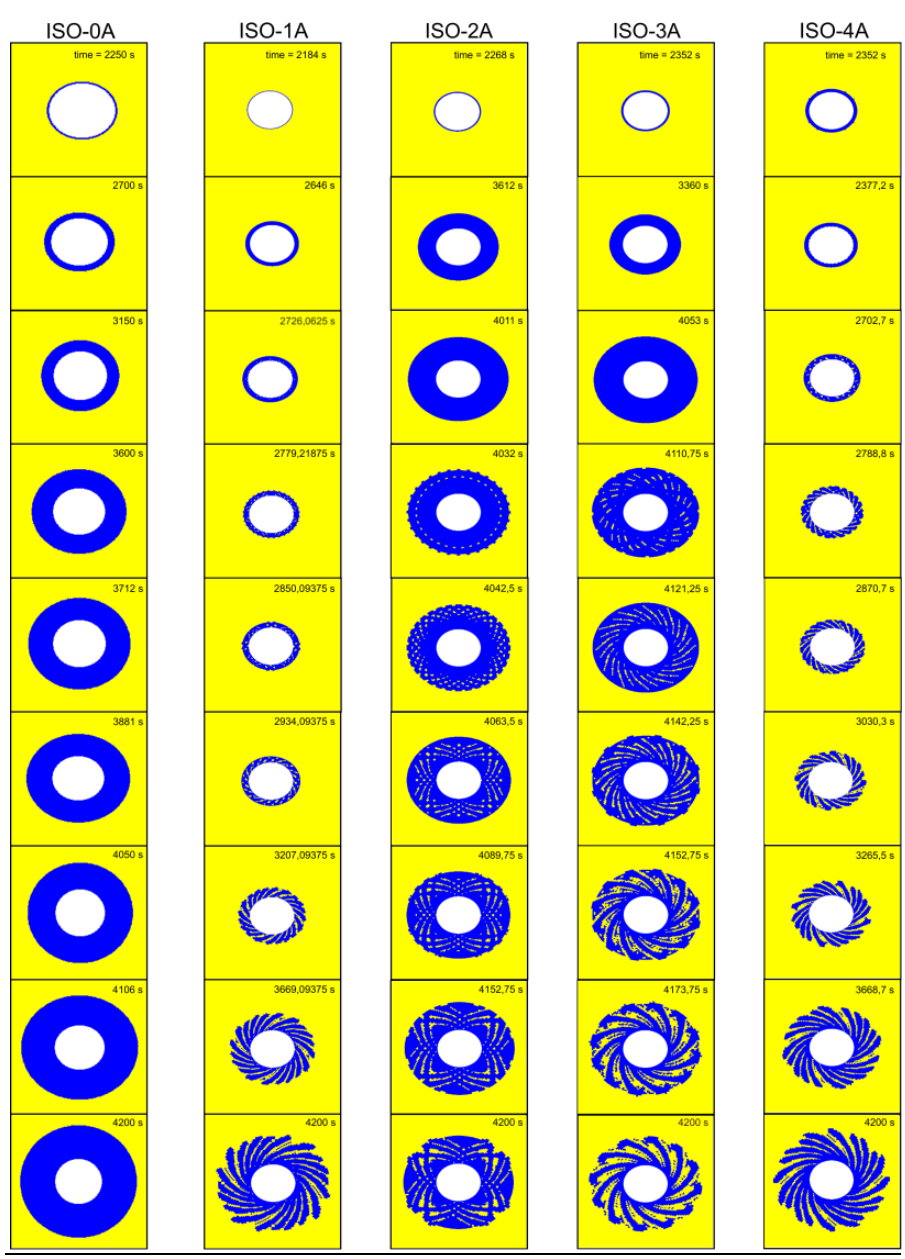

Fig. 2.Loading index of five solutions during phase A of boundary conditions

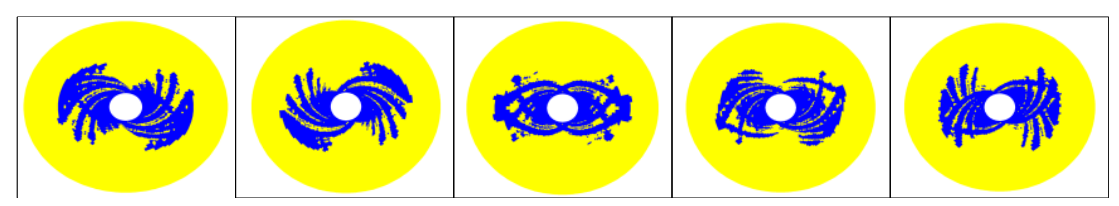

Fig. 3. Loading index at the end of the phase B

\section{Conclusions}

In this paper, the different solutions of the same initial boundary value problem (hollow cylinder experiment) for a fully saturated porous medium, modelled with an elastoplastic model, have been studied. All solutions have been obtained 
changing the time discretization of the problem. The solutions obtained with Plasol models can be classified according to the evolution of shear bands configurations in time.

It can be concluded from the present work that, as soon as degradation is incorporated in a constitutive equation, uniqueness of the solution of initial boundary value problems is questionable even in coupled problems. This work extends to the case of coupled problems the finding that the use of an enhanced second gradient model is not sufficient to restore the uniqueness of the solutions (see [4]).

\section{References}

[1] Hill R., Aspects of invariance in solid mechanics. Advances in applied mechanics 18, 1-75.

[2] Chambon R., Crochepeyre S. and Charilier R. 2001b. An algorithm and a method to search bifurcation points in non-linear problems. International Journal for Numerical Methods in Engineering 51 (3), 315-332.

[3] Collin, F., Chambon, R., Charlier, R., 2006. A finite element method for poro mechanical modelling of geotechnical problems using local second gradient models. International Journal for numerical methods in engineering 65, 1749-1772.

[4] Chambon R., Caillerie D., El Hassan N., 1998. One-dimensional localisation studied with asecond grade model. European Journal of Mechanics A/Solids 4, 637-656.

[5] Germain, P., November 1973b. The method of the virtual power in continuum mechanics. Part 2: Microstructure. Journal on Applied Mathematics 25 (3), 556-575.

[6] Barnichon, J.-D., 1998. Finite element modelling in structural and petroleum geology. Ph.D. thesis, Université de Liège Faculté des Sciences Appliquées.

[7] Ortiz, M., Simo, J. C., 1986. An analysis of a new class of integration algorithms for elastoplasticconstitutive relations. International Journal for Numerical Methods in Engineering 23 (3),353-366

[8] Mindlin, R. D., 1964. Micro-structure in linear elasticity. Archive for Rational Mechanics and Analysis, 51-78.

[9] Bésuelle, P., Chambon, R., Collin, F., 2006. Switching deformation modes in postlocalizationsolutions with quasi-brittle material. Mechanics of materials and structures 1 (7), $1115-1134$.

[10] Horseman, S. T., Winter, M. G., Entwistle, D. C., 1987. Geotechnical characterization of boomclay in relation to the disposal of radioactive waste (page 87). Tech. rep., Commission of theEuropean Communities, EUR 10987.

[11] Labiouse, V., Sauthier, C., You, S., 2013. Hollow cylinder simulation experiments of galleries inboom clay formation. Rock Mechanics and Rock Engineering, 1-13. 